\title{
FLUCTUACIONES DEL TIPO DE CAMBIO REALY TRANSABILIDAD DE BIENES EN EL COMERCIO BILATERAL CHILE-ESTADOS UNIDOS*
}

\author{
REAL EXCHANGE RATE FLUCTUATIONS AND TRADABILITY \\ OF GOODS IN CHILE-US INTERNATIONAL TRADE
}

\section{ANDRES SAGNERT.}

Banco Central de Chile

\begin{abstract}
Trade data shows that real exchange rate fluctuates more than other variables such as consumption and GDP, and also presents significant deviations from the Law of One Price. In light of these facts, this paper develops and estimates a non-monetary DSGE model under financial autarky for the international trade of goods and services between Chile and the U.S. In this context, the higher volatility of the real exchange rate is explained by the existence of transaction costs and varying degrees of imperfect substitution in consumption between home and foreign goods which endogenously induce a home bias effect. The results reveal that the estimated transaction costs and the asymmetric home bias effect content in the data would be responsible for the low correlation between consumption in both countries and the higher real exchange rate fluctuations. Finally, the model is able to replicate much of the stylized facts observed in the data, but fails to address the Backus-Smith puzzle.
\end{abstract}

Keywords: Real exchange rate, Law of One Price, tradability of goods, transaction costs, real business cycles.

JEL Classification: F11, F41.

* Agradezco los valiosos comentarios y sugerencias de Roberto Alvarez, Luis Opazo, Waldo Riveras, Javier Turén, los asistentes al Encuentro Anual 2009 de la Sociedad de Economía de Chile, un árbitro anónimo, y en especial de Rómulo Chumacero, en la elaboración de este artículo. Cualquier error es de mi exclusiva responsabilidad. E-mail: asagner@bcentral.cl 


\section{Resumen}

Los datos de comercio muestran que el tipo de cambio real fluctúa más que variables como el consumo y el producto y, además, presenta desvíos considerables de la Ley de Precio Unico. En vista de estos hechos, el artículo desarrolla y estima un modelo dinámico de equilibrio general estocástico no monetario y bajo autarquía financiera para el comercio internacional de bienes y servicios entre Chile y Estados Unidos. En este contexto, la mayor volatilidad del tipo de cambio real se explica por la existencia de costos de transacción y distintos grados de sustitución imperfecta entre el consumo de bienes nacionales y extranjeros que inducen endógenamente un sesgo hacia al consumo de bienes nacionales (sesgo local o home bias). Los resultados revelan que los costos de transacción estimados y el efecto home bias asimétrico contenido en los datos serían responsables de la baja correlación entre el consumo de ambos países y de las mayores fluctuaciones del tipo de cambio real. Finalmente, el modelo es capaz de replicar gran parte de los hechos estilizados observados en los datos, pero no logra solucionar el puzzle de Backus-Smith.

Palabras Clave: Tipo de cambio real, Ley de Precio Unico, transabilidad de bienes, costos de transacción, ciclos reales internacionales.

Clasificación JEL: F11, F41.

\section{INTRODUCCION}

La literatura define al tipo de cambio real como el precio relativo de bienes transables a no transables y, por lo tanto, la volatilidad de dicha variable se explicaría únicamente por movimientos en el precio relativo entre ambos bienes. Sin embargo, Engel (1999) y una gran cantidad de estudios empíricos posteriores muestran que el tipo de cambio real presenta fluctuaciones mayores que el consumo y el producto, $\mathrm{y}$ una proporción importante de estas variaciones es consecuencia de movimientos en el precio relativo de bienes transables, es decir, de desvíos desde la Ley de Precio Unico ${ }^{1}$. A la luz de esta evidencia, los modelos de ciclos reales internacionales han buscado explicar la volatilidad del tipo de cambio real de acuerdo a diversas especificaciones. Backus, Kehoe y Kydland (1994), por ejemplo, consideran mercados financieros completos y muestran que el tipo de cambio real de equilibrio es proporcional al ratio de consumo entre países. No obstante, su especificación falla en replicar la alta

1 Engel (1999) encuentra que más del 90\% de las fluctuaciones observadas en el tipo de cambio real entre varios países de la OECD y Estados Unidos pueden ser atribuidas a la variabilidad del precio relativo de transables. 
volatilidad de esta variable, puesto que bajo mercados completos los agentes pueden cubrirse totalmente del riesgo específico a cada país (risk sharing), lo que induce una correlación elevada y positiva entre el consumo de países, y finalmente, el ratio de consumo y el tipo de cambio real fluctúan poco. Esta discusión sugiere que la introducción de fricciones en el mercado internacional de activos puede ayudar a resolver esta anomalía. Así, Baxter y Crucini (1995), Kollman (1996) y Arvantis y Mikkola (1996) emplean modelos en los cuales el único activo transado internacionalmente es un bono no contingente. Los autores encuentran que tal especificación replica las altas fluctuaciones del tipo de cambio real sólo si los shocks de productividad son persistentes y si ellos no se extienden entre países.

Este artículo estudia las implicancias de una especificación alternativa, basada en los trabajos de Cole y Obstfeld (1991), Betts y Kehoe (2001) y Heathcote y Perri (2002), para explicar las fluctuaciones del tipo de cambio real entre Chile y Estados Unidos; aspecto que, a pesar de su relevancia para una economía pequeña y abierta como es el caso de Chile, cuenta con pocos estudios que lo abordan y estudian en detalle. En la especificación propuesta, la alta volatilidad de la variable se explicaría por dos aspectos clave del modelo. En primer lugar, la inexistencia del comercio internacional de activos financieros (autarquía financiera) imposibilita la cobertura de riesgo por parte de los agentes. Como consecuencia, la relación entre el tipo de cambio real y el ratio de consumo entre países desaparece. En segundo lugar, la clasificación dicotómica tradicional de bienes en transables o no transables sería inapropiada, puesto que ella se encontraría determinada por características fundamentales y potencialmente testeable del comercio internacional como los costos de transacción y el grado de sustitución entre bienes domésticos y extranjeros: los primeros explicarían los desvíos desde la Ley de Precio Unico, mientras que los segundos inducirían una menor correlación del consumo entre países. De esta forma, el artículo desarrolla un modelo dinámico de equilibrio general estocástico multipaís y multisector, no monetario y bajo autarquía financiera, que incorpora las fuentes de fluctuación del tipo de cambio real antes nombradas. En particular, el modelo considera la determinación del tipo de cambio real bajo un escenario de autarquía financiera y la existencia de costos de transacción sectoriales como los citados por Obstfeld y Rogoff (2000), además de distintos grados de sustitución imperfecta entre bienes del mismo tipo pero producidos por distintos países, los cuales generan endógenamente un sesgo hacia el consumo de bienes domésticos (home bias effect).

El ejercicio realizado y estimado para los sectores primarios, manufacturas, y construcción y servicios asociados al comercio internacional entre Chile y Estados Unidos es capaz de replicar la mayor volatilidad del tipo de cambio real respecto de variables como el consumo, el producto y la balanza comercial. Este resultado se explica, principalmente, por la existencia de un efecto home bias asimétrico entre ambos países y por los desvíos sectoriales desde la Ley de Precio Unico que son consistentes con los datos; resultados que son confirmados por los experimentos numéricos realizados. Finalmente, el modelo replica gran parte de los signos de comovimiento entre las principales variables macroeconómicas estudiadas pero falla en replicar el signo negativo de la correlación entre el ratio de consumo y el tipo de 
cambio real observada en los datos, anomalía conocida comúnmente como el Puzzle de Backus-Smith.

El artículo se encuentra organizado de la siguiente forma. La Sección 2 revisa la teoría asociada a la determinación tradicional del tipo de cambio real, la cual es contrastada con los hechos estilizados observados en los datos del comercio bilateral Chile-Estados Unidos. En la Sección 3 se describe el modelo dinámico de equilibrio general estocástico empleado para explicar las fuentes de fluctuación del tipo de cambio real, mientras que la Sección 4 detalla su especificación y la estimación de sus parámetros. La Sección 5 presenta los principales resultados obtenidos a partir del análisis de sensibilidad realizado para distintos parámetros del modelo estimado, y finalmente, la Sección 6 concluye.

\section{HECHOS ESTILIZADOS}

En esta sección se revisan los supuestos tradicionales tras la determinación del tipo de cambio real, los cuales son luego contrastados con los hechos estilizados contenidos en los datos del comercio internacional entre Chile y Estados Unidos.

De acuerdo a la teoría, los bienes se clasifican en dos categorías excluyentes entre sí: transables y no transables. El precio de equilibrio de los primeros es determinado por las fuerzas del comercio internacional y satisface la Ley de Precio Unico, mientras que el precio de los segundos se determina por condiciones internas del país que los produce. En este contexto y en cualquier período $t$, el tipo de cambio real entre Chile y Estados Unidos $R E R_{t}$ se encuentra determinado por:

$$
R E R_{t}=N E R_{t}\left(\frac{P_{t}^{U S}}{P_{t}^{C H}}\right)
$$

donde $N E R_{t}$ es el tipo de cambio nominal expresado en pesos chilenos por dólar, y $P_{t}^{U S}$ y $P_{t}^{C H}$ es un índice de precios agregado para Estados Unidos y Chile, respectivamente. Los elementos de la ecuación (1) son una clara consecuencia de los supuestos relacionados con la transabilidad dicotómica de bienes que impone la teoría tradicional: $N E R_{t}$ mide el precio relativo de bienes transables, mientras que el cuociente $\left(P_{t}^{U S} / P_{t}^{C H}\right)$ mide el precio relativo asociado a bienes no transables. Adicionalmente, la Ley de Precio Unico señala que si ambos países producen exactamente la misma canasta de bienes transables en cada período $t$, entonces el precio de transables en Chile $P T_{t}^{C H}$ será igual al precio de su contraparte en Estados Unidos $P T_{t}^{U S}$, expresado en pesos chilenos, es decir:

$$
P T_{t}^{C H}=N E R_{t} \cdot P T_{t}^{U S}
$$

A partir de la ecuación (2), $R E R_{t}$ puede ser reescrito como: 


$$
R E R_{t}=\left[N E R_{t}\left(\frac{P T_{t}^{U S}}{P T_{t}^{C H}}\right)\right] \cdot\left[\left(\frac{P T_{t}^{C H}}{P T_{t}^{U S}}\right)\left(\frac{P_{t}^{U S}}{P_{t}^{C H}}\right)\right]
$$

En la expresión anterior, $R E R_{t}=N E R_{t}\left(P T_{t}^{U S} / P T_{t}^{C H}\right)$ corresponde a la porción del tipo de cambio real que es explicada por el precio relativo de bienes transables expresado en términos de una moneda común $\left(\widetilde{R E R}_{t}\right)$, mientras que el producto $\left(P T_{t}^{C H} / P T_{t}^{U S}\right) \cdot\left(P_{t}^{U S} / P_{t}^{C H}\right)$ es la porción del tipo de cambio real atribuible al precio relativo de bienes no transables $\left(\widehat{R E R}_{t}\right)$, la cual es denominada por Betts y Kehoe (2001, 2008) como tipo de cambio real explicado. De este modo:

$$
R E R_{t}=\widehat{R E R}_{t} \cdot \widehat{R E R_{t}}
$$

De acuerdo a los supuestos anteriores, la correlación entre $R E R_{t}$ y $\widetilde{R E R}_{t}$ es igual a cero, y por lo tanto, las fluctuaciones del tipo de cambio real son sólo consecuencia de movimientos en $\widehat{R E R}_{t}{ }^{2}$. No obstante lo anterior, una cantidad considerable de estudios empíricos han reportado correlaciones positivas y significativas entre el tipo de cambio real y el precio relativo de bienes transables ${ }^{3}$.

Una hipótesis que ayudaría a explicar esta anomalía en la determinación del tipo de cambio real postula que el producto de distintos sectores, en lugar de ser perfectamente transable o completamente no transable, poseería distintos grados de transabilidad, los cuales estarían determinados por características fundamentales y potencialmente tratables del comercio internacional: costos de transacción y el grado de sustitución imperfecta en el consumo de bienes domésticos y extranjeros del mismo tipo (producidos por el mismo sector). La consideración de los primeros se fundamenta sobre el trabajo de Obstfeld y Rogoff (2000). En su artículo, los autores indican que los costos de transacción pueden ser pensados como aranceles, costos de transporte, costos legales y administrativos, y/o barreras no arancelarias al comercio internacional ${ }^{4}$. Así, los distintos grados de transabilidad de bienes son proporcionales a las magnitudes de los costos de transacción involucrados ${ }^{5}$. La consideración de los distintos grados de sustitución imperfecta entre bienes domésticos y extranjeros, por

2 De acuerdo a la Ley de Precio Unico $\widetilde{R E R}_{t}=1 \mathrm{y}$, por lo tanto, $\operatorname{corr}\left(R E R_{t}, \widetilde{R E R}_{t}\right)=0$. De esta forma, la comparación de las propiedades de series de tiempo entre $R E R_{t}$ y $\widehat{R E R}_{t}$ es una útil herramienta para dilucidar la validez de los supuestos que subyacen la teoría tradicional.

3 Ver por ejemplo; Lipsey y Kravis (1978); Engel (1993); Lapham (1995); Engel y Rogers (1996); Knetter (1997); Engel (1999); Betts y Devereux (2000); Chari, Kehoe y McGrattan (2001); y Betts y Kehoe (2001, 2008), entre muchos otros.

4 Si bien la entrada en vigencia del Tratado de Libre Comercio entre Chile y Estados Unidos en enero de 2004 haría pensar que dichos costos han tendido a disiparse, el contenido del documento estipula sólo la eliminación paulatina de barreras arancelarias entre ambos países.

5 En este contexto, los bienes transables son aquellos que poseen costos de transacción igual a cero, mientras que los bienes no transables poseen costos de transacción infinitos. 
su parte, se basa sobre los trabajos de McCallum (1995) y Helliwell (1996), los cuales encuentran evidencia de efectos "frontera" sorprendentemente grandes en el comercio internacional entre Estados Unidos y Canadá6. De esta forma, bajo el supuesto que la intensidad con la cual un determinado bien es transado reflejaría exactamente su grado de transabilidad (Betts y Kehoe, 2001), el volumen de comercio entre dos países reflejaría la tasa a la cual los agentes están dispuestos a sustituir bienes importados por bienes domésticos ${ }^{7}$.

De este modo, se construyeron medidas para $R E R_{t}$ y $\widehat{R E R}_{t}$ mediante índices de precios de bienes transables e índices de precios agregados de Chile y Estados Unidos. En particular, se recolectaron datos en frecuencia anual del período 1987 a 2007 para tres sectores productivos: agricultura, pesca y minería (en adelante primarios); manufacturas; y construcción y servicios ${ }^{8}$. La elección de estos sectores productivos responde a dos razones básicas. Primero, ante la presencia de distintos grados de transabilidad dichos sectores son conceptualmente más fáciles de entender como transables o no transables. Segundo, y como se verá en detalle en la próxima sección, la incorporación de sectores productivos adicionales incrementa de forma importante las ecuaciones y parámetros del modelo.

El primer paso en la construcción de un índice de precios de transables consistió en la recolección de datos del comercio bilateral entre ambos países, detallados para los tres sectores descritos, con el fin de elaborar un índice de transabilidad sectorial para Chile $I T_{j}^{C H}$ dado por:

$$
I T_{j}^{C H}=100 \cdot\left[\frac{1}{21} \sum_{t=1987}^{2007}\left(\frac{X_{j, t}^{C H}+M_{j, t}^{C H}}{Y_{j, t}^{C H}}\right)\right], \quad j=\{\text { pri, man }, \text { cys }\}
$$

donde $X_{j, t}^{C H}, M_{j, t}^{C H}$ e $Y_{j, t}^{C H}$ corresponden a las exportaciones, importaciones y el producto chileno del sector $j$, respectivamente. Este índice permitirá identificar aquellos sectores que producen bienes relativamente más transables y, por lo tanto, identificar los sectores que se incluirán en el índice de precios de bienes transables.

6 McCallum (1995) encuentra que el comercio interestatal de Estados Unidos es cerca de veinte veces mayor que aquel observado entre estados de Canadá y Estados Unidos. Helliwell (1996) confirma los resultados de McCallum utilizando datos similares e incluso focalizando el análisis sólo a Quebec.

7 Isard (1977), Giovannini (1988) y Engel y Rogers (1996) sugieren que los distintos grados de transabilidad de bienes podrían deberse también a problemas de agregación. A un nivel retail, muchos bienes clasificados como transables incorporan una gran cantidad de componentes no transables. En consecuencia, el precio de bienes "supuestamente" transables se encontraría tan aislado de las fuerzas del arbitraje internacional como el precio de no transables.

8 Los datos fueron obtenidos desde el Banco Central de Chile, Bureau of Economic Analysis y la base de datos Direction of Trade del Fondo Monetario Internacional. El detalle se encuentra en el Anexo A.1 del documento. 
TABLA 1

INDICE DE TRANSABILIDAD EN CHILE

1987-2007 (\%)

\begin{tabular}{|lcc|}
\hline Sector $(j)$ & $I T_{j}^{C H}$ & $\sigma_{I T}^{C H}$ \\
\hline Primario & 17,34 & 3,29 \\
Manufacturas & 29,29 & 2,70 \\
Construcción y Servicios & 6,66 & 0,94 \\
\hline
\end{tabular}

La Tabla 1 reporta el comercio total entre ambos países como porcentaje del producto sectorial de Chile durante el período 1987-2007. Claramente, el grado de transabilidad varía de forma considerable y estadísticamente significativa entre sectores: el sector manufacturas es el más transado, seguido por el sector primario, y, finalmente, por construcción y servicios. Más aún, la magnitud de la desviación estándar reportada en la tercera columna de la tabla anterior rechaza la hipótesis nula $H_{0}: I T_{j}^{C H}=0,\left(H_{0}: I T_{j}^{C H}=100\right), j=\{$ pri,man,$c y s\}$, para niveles de significancia estándares. A partir de estos resultados y siguiendo la literatura de comercio internacional, se supuso que el sector construcción y servicios es no transable, mientras que los sectores primarios y manufacturas son transables. Así, bajo este supuesto, el índice de precios de bienes transables $P T_{j}^{i}$ se calculó a partir del deflactor del producto y se encuentra dado por?:

$$
P T_{j}^{i}=\frac{Y_{p r i, t}^{i}+Y_{m a n, t}^{i}}{\tilde{Y}_{p r i, t}^{i}+\tilde{Y}_{m a n, t}^{i}}, \quad i=\{C H, U S\}
$$

donde $Y_{j, t}^{i}$ e $\tilde{Y}_{j, t}^{i}$ corresponden al producto nominal y real del sector $j$ que opera en el país $i$, respectivamente. El índice de precios agregados $P_{t}^{i}$ fue calculado de forma análoga como:

$$
P_{t}^{i}=\frac{Y_{p r i, t}^{i}+Y_{m a n, t}^{i}+Y_{c y s, t}^{i}}{\tilde{Y}_{p r i, t}^{i}+\tilde{Y}_{m a n, t}^{i}+\tilde{Y}_{c y s, t}^{i}}, \quad i=\{C H, U S\}
$$

9 Betts y Kehoe (2005) argumentan que el deflactor del producto es una medida más adecuada que un índice de precios de transables obtenido a partir del valor agregado, debido a que el primero mide el valor de la producción en el sitio donde ella se lleva a cabo y, por lo tanto, excluye el valor de ciertos servicios no transables. El segundo, en contraste, excluye el precio de algunos bienes intermedios transables. 
En la Figura 1 se muestran las series de $R E R_{t}$ y $\widehat{R E R}_{t}$ construidas a partir de las definiciones anteriores. De ella se puede apreciar que el tipo de cambio real presenta una mayor volatilidad con respecto al tipo de cambio real explicado. En efecto, la desviación estándar de $R E R_{t}$ es 2,59 veces mayor que aquella de $\widehat{R E R}_{t}$. En adición, la correlación entre ellas es prácticamente nula e igual a 0,04 , lo que indica que la trayectoria de ambas series no presenta un patrón de comovimiento común. Este simple análisis de series de tiempo revela la existencia de desviaciones considerables desde la Ley de Precio Unico para las canastas de bienes empleadas en la construcción del índice de precios transables.

\section{FIGURA 1}

TIPO DE CAMBIO REAL CHILE-ESTADOS UNIDOS

(Base: 1987=100)

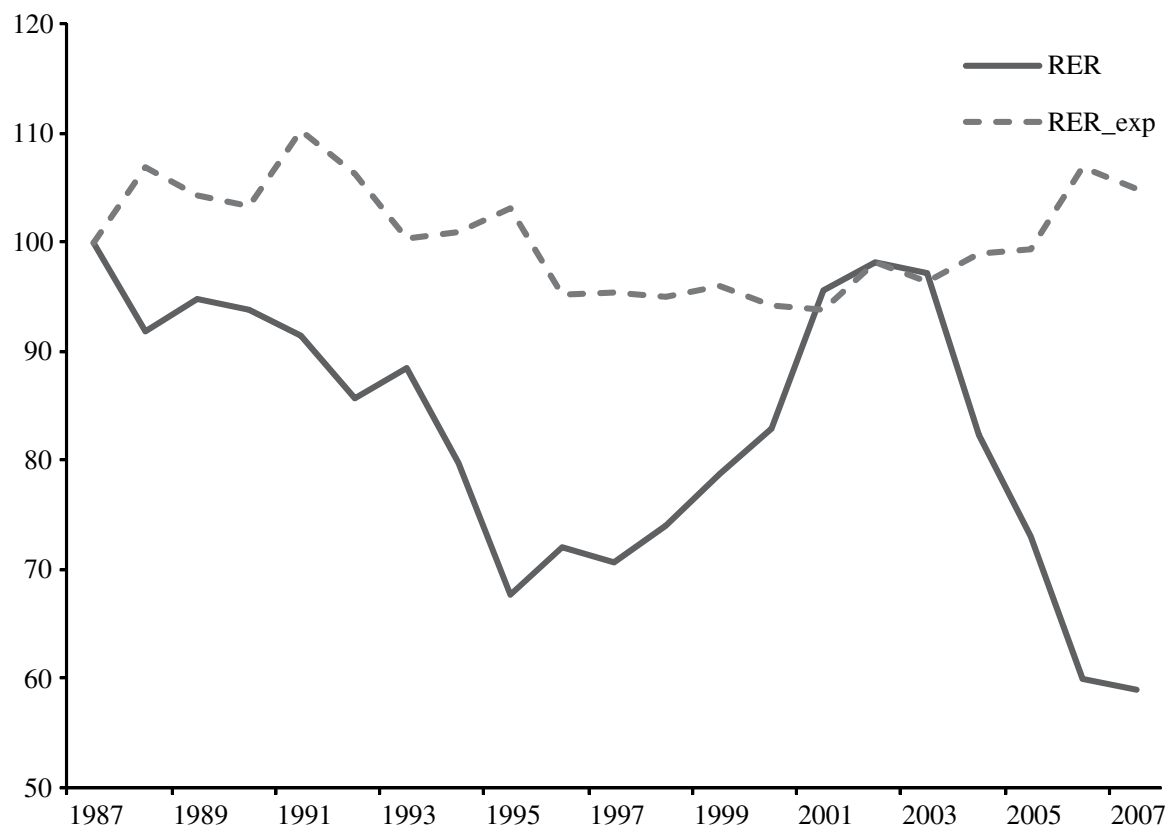


TABLA 2

DESVIACIONES SECTORIALES LEY DE PRECIO UNICO 1987-2007 (\%)

\begin{tabular}{|llc|}
\hline Sector $(j)$ & $D_{j}^{L P U}$ & $I C_{95 \%}$ \\
\hline Primario & 27,72 & {$[21,32 ; 39,61]$} \\
Manufacturas & 20,32 & {$[15,63 ; 29,05]$} \\
Construcción y Servicios & 16,58 & {$[12,75 ; 23,69]$} \\
\hline
\end{tabular}

En la segunda columna de la Tabla 2 se reporta las desviaciones sectoriales desde la Ley de Precio Unico $D_{j}^{L P U}$ calculadas como:

$$
D_{j}^{L P U}=100 \cdot\left[\frac{1}{21} \sum_{t=1987}^{2007}\left(\log \widetilde{R E R}_{j, t}-\log \overline{\widetilde{R E R}}_{j}\right)^{2}\right]^{1 / 2}, \quad j=\{\text { pri, man }, \text { cys }\}
$$

donde $\widetilde{\widetilde{R E R}}_{j}$ es el promedio de $\widetilde{R E R}_{j, t}$ durante el período 1987-2007. De acuerdo a la ecuación (8), si la Ley de Precio Unico se cumpliese para algún sector $j$, entonces $D_{j}^{L P U}$ debiese ser igual a cero. Los resultados de la Tabla 2 muestran, por el contrario, que las desviaciones para aquellos sectores definidos como transables -primario y manufacturas- varían ampliamente y el intervalo al 95\% de confianza de este estimador indica que ninguna de ellas es estadísticamente igual a cero.

En resumen, la evidencia contenida en los datos del comercio bilateral entre Chile y Estados Unidos rechaza los dos supuestos que subyacen en la determinación tradicional del tipo de cambio real. En primer lugar, los índices de transabilidad sectoriales, estadísticamente distintos de $0 \%$ y $100 \%$ exhibidos en la Tabla 1, apoyan la hipótesis de que los bienes no son perfectamente transables o no transables. En segundo lugar, las fluctuaciones del tipo de cambio real explicado son considerablemente menores que aquellas observadas en el tipo de cambio real y la correlación entre ambas series es prácticamente nula, hechos que se ven reflejados en los desvíos sectoriales desde la Ley de Precio Unico reportados en la Tabla 2. Estas regularidades son la motivación para el desarrollo de un modelo de equilibrio general estocástico en la próxima sección, el cual incorpora costos de transacción y distintos grados de sustitución imperfecta entre bienes domésticos y extranjeros como fuente de los distintos grados de transabilidad y, por ende, de la mayor volatilidad de $R E R_{t}$ respecto de $\widehat{R E R}_{t}$. El modelo incorpora detalles a nivel sectorial y es explícitamente cuantitativo, en el sentido que de él se pueden construir series de $R E R_{t}$ y $\widehat{R E R}_{t}$ similares a las enunciadas en esta sección. 


\section{EL MODELO}

En esta sección se presenta el modelo dinámico de equilibrio general estocástico bajo autarquía financiera e inexistencia de dinero empleado en este artículo, el cual se basa en el modelo multipaís y multisector desarrollado por Betts y Kehoe (2001). La especificación original de los autores es modificada en tres aspectos principales: (i) el modelo es dinámico, en el sentido de que incorpora una ley de movimiento para la acumulación de capital sectorial; (ii) se considera shocks de productividad sectoriales en lugar de shocks de preferencias; y (iii) la especificación incluye elasticidades de sustitución entre bienes domésticos y extranjeros sectoriales y específicas a cada país.

El mundo se encuentra conformado por $I$ países, cada uno de los cuales posee $J$ sectores productivos que elaboran un único tipo de bien. En cada país habita un consumidor representativo que vive infinitos períodos y que consume los $J$ tipos de bienes producidos por los $I$ países. En consecuencia, el producto de un determinado sector producido por dos países distintos es visto como sustituto imperfecto en el consumo, existiendo así $I \times J$ bienes diferenciados en la economía mundial. Los bienes diferenciados se encuentran adicionalmente caracterizados por costos de transacción, los cuales serán descritos más adelante.

La notación empleada es la siguiente: los países de origen son indexados por $h=1, \ldots, I$, los países de destino por $i=1, \ldots, I$ y los sectores productivos por $j=1, \ldots, J$. Por otra parte, subíndices denotan países y sectores de origen, mientras que superíndices indican países de destino. De esta forma, $c_{j, h}^{i}$ denota el consumo en el país $i$ del bien $j$ producido por el país $h$.

\subsection{Hogares}

El consumidor representativo del país $i, i=1, \ldots, I$, dispone de 1 unidad perfectamente divisible de tiempo, la cual destina a trabajo y ocio. Dado que el trabajo es inmóvil entre países, la fracción de tiempo destinada a trabajar puede ser repartida sólo entre los $J$ sectores productivos que operan en el país $i$. El problema de optimización enfrentado por el consumidor representativo $i$ se encuentra dado por:

$$
\max E_{t} \sum_{t=0}^{\infty} \beta^{t} u^{i}\left(c_{t}^{i}, 1-l_{t}^{i}\right), \quad i=1, \ldots, I
$$

donde $\beta$ es el factor de descuento intertemporal y la utilidad instantánea $u^{i}(\cdot)$ es función de la canasta de consumo $c_{t}^{i}$ y del ocio $\left(1-l_{t}^{i}\right)$, y se asume monotónica, creciente, estrictamente cóncava y $\lim _{c_{t}^{i} \rightarrow 0}\left(\partial u^{i} / \partial c_{t}^{i}\right)=\infty$. La canasta de consumo $c_{t}^{i}$, por su parte, corresponde a un agregador tipo CES sobre el consumo de canastas 
sectoriales $c_{j, t}^{i}$, el cual se asume creciente, monotónico y homogéneo de grado uno, y se encuentra dado por:

$$
c_{t}^{i}=\left[\sum_{j=1}^{J} \varsigma_{j}^{i}\left(c_{j, t}^{i}\right)^{\psi^{i}}\right]^{1 / \psi^{i}}, \quad i=1, \ldots, I
$$

En la ecuación anterior, los parámetros $\varsigma_{j}^{i}$ recogen las preferencias del consumidor representativo $i$ por las distintas canastas sectoriales $j$, mientras que el parámetro $\psi^{i}$ es igual a 1 menos el inverso de la elasticidad de sustitución entre canastas sectoriales consumidas en el país $i$. De forma análoga, $c_{j, t}^{i}$ corresponde a un agregador tipo CES sobre el consumo de bienes domésticos $c_{j, h, t}^{h}$ y extranjeros $c_{j, h, t}^{i}$ :

$$
c_{j, t}^{i}=\left[\sum_{h=1}^{I} \alpha_{h}^{i}\left(c_{j, h, t}^{i}\right)^{\rho_{j}^{i}}\right]^{1 / \rho_{j}^{i}}, \quad i=1, \ldots, I, \quad j=1, \ldots, J
$$

donde los parámetros $\alpha_{h}^{i}$ representan las preferencias del consumidor representativo $i$ por bienes producidos internamente, $h=i$, e importaciones, $h \neq i$ (es decir, los parámetros $\alpha_{h}^{i}$ determinan el grado de sesgo hacia el consumo de bienes domésticos o home bias effect), y $\rho_{j}^{i}$ es igual a 1 menos el inverso de la elasticidad de sustitución entre bienes del sector $j$ consumidos en el país $i$.

En cada período $t$, el agente representativo $i$ percibe ingresos $w_{t}^{i} l_{t}^{i}$ por trabajar e ingresos $r_{t}^{i} k_{t}^{i}$ por la renta del stock de capital a las firmas, donde $w_{t}^{i} \mathrm{y} r_{t}^{i}$ corresponden al salario e interés nominal, respectivamente. Como el consumidor representativo es también dueño de las firmas que producen los $J$ tipos de bienes, él recibe igualmente los beneficios $\pi_{j, i, t}$ de cada firma que opera en el sector productivo $j$. El ingreso total es destinado al consumo de los $J$ tipos de bienes nacionales e importados desde cada país $h$, y a la acumulación de capital $i_{t}^{i}$, el cual se deprecia a una tasa $\delta$. Así, la restricción presupuestaria enfrentada por el agente es la siguiente:

$$
\begin{gathered}
\sum_{j=1}^{J} \sum_{h=1}^{I}\left(1+\tau_{j, h}^{i}\right) p_{j, h, t} c_{j, h, t}^{i}+i_{t}^{i}=w_{t}^{i} l_{t}^{i}+r_{t}^{i} k_{t}^{i}+\sum_{j=1}^{J} \pi_{j, i, t}, \quad i=1, \ldots, I \\
i_{t}^{i}=k_{t+1}^{i}-(1-\delta) k_{t}^{i}, \quad i=1, \ldots, I
\end{gathered}
$$

donde $p_{j, h, t}$ corresponde al precio asociado al consumo del bien $j$ producido en el país $h$ y el precio de la inversión fue normalizado a 1 . Los costos de transacción $\tau_{j, h}^{i}$ 
se asumen proporcionales al número de unidades comercializadas (Obstfeld y Rogoff, 2000). Específicamente, si una unidad del bien $j$ es exportada desde el país $h$ al país $i$, entonces $\tau_{j, h}^{i}$ unidades de este bien se pierden en el proceso de exportación ${ }^{10}$.

En resumen, el problema del consumidor representativo $i$ consiste en maximizar (9) sujeto a las restricciones (10) a (13).

\subsection{Firmas}

En cada período $t$, el bien tipo $j$ es producido por una firma representativa que opera en el país $h$ y que maximiza beneficios según:

$$
\max \quad \pi_{j, h, t}=p_{j, h, t} y_{j, h, t}-w_{t}^{h} l_{j, t}^{h}-r_{t}^{h} k_{j, t}^{h}, \quad h=1, \ldots, I, \quad j=1, \ldots, J
$$

donde $y_{j, h, t}$ es el producto del sector $j$ obtenido a partir de una tecnología de producción $f(\cdot)$ estrictamente cóncava. La tecnología $f(\cdot)$ es función del stock del capital $k_{j, t}^{h}$ y del trabajo $l_{j, t}^{h}$-ambos inmóviles internacionalmente- empleados en dicho sector, además de un shock de productividad sectorial $z_{j, h, t}$ :

$$
y_{j, h, t}=f\left(k_{j, t}^{h}, l_{j, t}^{h}, z_{j, h, t}\right), \quad h=1, \ldots, I, \quad j=1, \ldots, J
$$

El problema de optimización enfrentado por la firma representativa $i$ consiste entonces en maximizar (14) sujeto a la tecnología de producción dada por (15).

\subsection{Equilibrio Interno}

En cada período $t$, los mercados se vacían, es decir:

$$
\begin{gathered}
\sum_{i=1}^{I}\left(1+\tau_{j, h}^{i}\right) c_{j, h, t}^{i}+i_{j, t}^{h}=y_{j, h, t}, \quad h=1, \ldots, I, \quad j=1, \ldots, J \\
i_{j, t}^{h}=k_{j, t+1}^{h}-(1-\delta) k_{j, t}^{h}, \quad h=1, \ldots, I, \quad j=1, \ldots, J
\end{gathered}
$$

10 De esta definición es claro que $\tau_{j, h}^{i}=0$ para todo $h=i$. 


$$
\begin{aligned}
& \sum_{j=1}^{J} l_{j, t}^{h}=l_{t}^{h}, \quad h=1, \ldots, I \\
& \sum_{j=1}^{J} k_{j, t}^{h}=k_{t}^{h}, \quad h=1, \ldots, I
\end{aligned}
$$

La ecuación (16) indica que el producto del sector $j$ elaborado en el país $h$ es destinado al consumo de bienes domésticos, exportaciones y a la acumulación de capital sectorial (17). Las ecuaciones (18) y (19), por su parte, resumen el cierre de los mercados de factores en cada país $h$.

\subsection{Equilibrio Mundial}

En cada período $t$, el equilibrio mundial del modelo se encuentra empleando las ecuaciones (12) - (14) y (16) - (19), y se halla dado por:

$$
\sum_{h=1}^{I}\left(c_{j, h, t}^{h}+i_{j, t}^{h}\right)=\sum_{h=1}^{I} y_{j, h, t}, \quad j=1, \ldots, J
$$

\subsection{El Problema del Planificador Mundial}

La teoría de equilibrio general postula que, ante la ausencia de distorsiones como impuestos o subsidios, el equilibrio asociado a la representación descentralizada de esta economía es equivalente a aquel obtenido a partir del problema enfrentado por un planificador mundial ${ }^{11}$. Así, dado un vector de ponderadores $a=\left(a^{1}, \ldots, a^{I}\right)$, el problema corresponde a:

$$
\begin{gathered}
\max E_{t}\left[\sum_{i=1}^{I} a^{i} \sum_{t=0}^{\infty} \beta^{t} u^{i}\left(c_{t}^{i}, 1-l_{t}^{i}\right)\right], \quad i=1, \ldots, I \\
\text { s.a. } \sum_{i=1}^{I}\left(1+\tau_{j, h}^{i}\right) c_{j, h, t}^{i}+i_{j, t}^{h}=f\left(k_{j, t}^{h}, l_{j, t}^{h}, z_{j, h, t}\right), \quad h=1, \ldots, I, \quad j=1, \ldots, J \\
i_{j, t}^{h}=k_{j, t+1}^{h}-(1-\delta) k_{j, t}^{h}, \quad h=1, \ldots, I, \quad j=1, \ldots, J
\end{gathered}
$$

11 Esta equivalencia será utilizada en el presente artículo como una forma de simplificar la resolución del modelo. La demostración de ella se encuentra en el Anexo A.2 del documento. 


$$
\begin{gathered}
\sum_{j=1}^{J} l_{j, t}^{h}=l_{t}^{h}, \quad h=1, \ldots, I \\
c_{t}^{i}=\left[\sum_{j=1}^{J} \varsigma_{j}^{i}\left(c_{j, t}^{i}\right)^{\psi^{i}}\right]^{1 / \psi^{i}}, \quad i=1, \ldots, I \\
c_{j, t}^{i}=\left[\sum_{h=1}^{I} \alpha_{h}^{i}\left(c_{j, h, t}^{i}\right)^{\rho_{j}^{i}}\right]^{1 / \rho_{j}^{i}}, \quad i=1, \ldots, I, \quad j=1, \ldots, J
\end{gathered}
$$

En este contexto, los precios $p_{j, h, t}, w_{t}^{h}$ y $r_{t}^{h}$ se encuentran definidos por:

$$
\begin{gathered}
p_{j, h, t}\left(a, z_{j, h, t}\right)=a^{h} \frac{\partial u^{h}\left(c_{t}^{h}, 1-l_{t}^{h} ; z_{j, h, t}\right)}{\partial c_{j, h, t}^{h}}, \quad h=1, \ldots, I, \quad j=1, \ldots, J \\
w_{t}^{h}\left(a, z_{j, h, t}\right)=a^{h} \frac{\partial f\left(k_{j, t}^{h}, l_{j, t}^{h}, z_{j, h, t}\right)}{\partial l_{j, t}^{h}}, \quad h=1, \ldots, I, \quad j=1, \ldots, J \\
r_{t}^{h}\left(a, z_{j, h, t}\right)=a^{h} \frac{\partial f\left(k_{j, t}^{h}, l_{j, t}^{h}, z_{j, h, t}\right)}{\partial k_{j, t}^{h}}, \quad h=1, \ldots, I, \quad j=1, \ldots, J
\end{gathered}
$$

La ecuación (22) indica que en el equilibrio, el precio del bien doméstico $j$ que es consumido en su país de origen es simplemente la utilidad marginal asociada al consumo de él, ponderada por el peso que el planificador mundial le atribuye a las preferencias del consumidor representativo $h$, mientras que el salario y el interés nominal corresponden a la productividad marginal del trabajo (23) y del capital (24) ponderadas por el peso $a^{h}$, respectivamente. 


\section{ESPECIFICACION Y ESTIMACION DEL MODELO}

En esta sección se detalla el modo como las preferencias, la tecnología de producción y la ley de movimiento de los shocks de productividad sectoriales fueron especificados. Finalmente, se presentan los resultados de la estimación de los parámetros del modelo.

\subsection{Especificación}

La versión del modelo empleada en este artículo considera un mundo conformado por dos países -Chile $(\mathrm{CH})$ y Estados Unidos $(U S)$ - donde ambos poseen sólo tres sectores productivos: primarios, manufacturas, y construcción y servicios (sectores 1,2 y 3 , respectivamente). Sobre esta base, la función de utilidad instantánea $u^{i}($. adopta una forma del tipo King, Plosser y Rebelo (1988), y se encuentra dada por:

$$
u^{i}\left(c_{t}^{i}, 1-l_{t}^{i}\right)=\frac{\left[\left(c_{t}^{i}\right)^{\eta}\left(1-l_{t}^{i}\right)^{1-\eta}\right]^{1-\gamma}}{1-\gamma}, \quad i=\{C H, U S\}
$$

donde $\gamma$ es un parámetro que resume la aversión relativa al riesgo por parte de los agentes y $\eta$ es una medida para el grado de sustitución entre consumo y ocio.

La tecnología de producción $f(\cdot)$, por su parte, es del tipo Cobb-Douglas con rendimientos constantes a escala:

$$
f\left(k_{j, t}^{h}, l_{j, t}^{h}, z_{j, h, t}\right)=A_{j, h} e^{z_{j, h, t}}\left(k_{j, t}^{h}\right)^{\theta_{j, h}}\left(l_{j, t}^{h}\right)^{1-\theta_{j, h}}, \quad i=\{C H, U S\}, \quad j=1,2,3
$$

donde $\theta_{j, h}$ es la participación del capital en el producto del sector $j$, y $A_{j, h}$ es un parámetro de escala.

Por último, la ley de movimiento de los shocks de productividad sectoriales se encuentra gobernada por un proceso autorregresivo de orden 1 estacionario:

$$
z_{j, h, t}=\phi_{j, h} z_{j, h, t-1}+\varepsilon_{j, h, t}, \quad\left|\phi_{j, h}\right|<1, \quad h=\{C H, U S\}, \quad j=1,2,3
$$

donde $\varepsilon_{j, h, t} \sim N\left(0, \sigma_{\varepsilon_{j, h}^{2}}^{2}\right), E\left[\varepsilon_{j, h, t} \varepsilon_{j, h, s}\right]=0, \forall t \neq s$, es ruido blanco. Por simplicidad, y siguiendo a Baxter y Crucini (1995), Kollman (1996), Arvantis y Mikkola (1996) y 
Betts y Kehoe (2001), la especificación no considera la existencia de efectos spillover en la ley de movimiento de los shocks de productividad sectoriales ${ }^{12}$.

\subsection{Estimación}

La especificación anterior implica que la versión del modelo con dos países y tres sectores productivos posee una cantidad considerable de parámetros fijos a estimar (54 en total), hecho que motivó un procedimiento de estimación por grupos.

En primer lugar, los parámetros $\beta, \gamma, \eta, \delta, \psi^{i}, \rho_{j}^{i}$ y $\theta_{j, i}$ fueron obtenidos desde la literatura de ciclos reales y se reportan en la Tabla 3. La elección del valor para el factor de descuento intertemporal $(0,95)$ responde a dos criterios. Primero, los datos empleados para la estimación de los parámetros del modelo se encuentran en frecuencia anual. Segundo, y más importante aún, Bodenstein (2006) muestra que si $\beta$ adopta valores bajos, es decir, los agentes son suficientemente impacientes, entonces el modelo es capaz de generar una mayor volatilidad del tipo de cambio real respecto de los principales agregados macroeconómicos ${ }^{13}$. Por otra parte, la aversión relativa al riesgo $\gamma$ es igual a 2, valor que se encuentra dentro del rango empleado en otros estudios donde el parámetro es usualmente asumido entre 1 y 6.

Los parámetros $\rho_{j}^{i}$ fueron obtenidos de Broda, Greenfield y Weinstein (2006). En su trabajo, los autores estiman vía GMM las elasticidades de sustitución sectorial entre bienes domésticos y extranjeros de 73 países durante el período 1994-2003, detalladas a un nivel de tres dígitos del Sistema Armonizado de Designación y Codificación de Mercancías (HS), lo que equivale a un total de 171 sectores productivos.

El segundo grupo de parámetros, conformado por $a^{i}, \alpha_{h}^{i}, \varsigma_{j}^{i}, \tau_{j, h}^{i}$ y $A_{j, i}$ fue estimado de forma tal que el estado estacionario del modelo replique los hechos estilizados observados en los datos (Tabla 4$)^{14}$.

12 El efecto spillover mide el impacto en la productividad doméstica (extranjera) relacionada a un cambio en la productividad extranjera (doméstica). La simplicidad de esta especificación proviene del hecho de que se podría considerar, alternativamente, un efecto spillover intrasectores (la productividad del sector doméstico $j$ afecta la productividad de su contraparte extranjera, y viceversa), o bien uno intersectores (la productividad del sector $j$ afecta la productividad de todos los sectores extranjeros, y viceversa), lo que en ambos casos incrementaría la cantidad de $\phi$ 's a estimar. Corsetti, Dedola y Leduc (2004) muestran que la no consideración de este efecto en modelos dinámicos de equilibrio general estocástico no afecta de forma sustancial la volatilidad del tipo de cambio real generada por él.

13 Bodenstein (2006) emplea un modelo con dos países y mercados financieros completos pero donde los contratos financieros internacionales son ejecutables sólo de forma limitada. Como consecuencia, los agentes pueden cubrirse parcialmente del riesgo específico a cada país (partial risk sharing). El autor muestra que para un factor de descuento intertemporal $\beta=0,95$, es decir, los agentes son suficientemente impacientes, los resultados de su modelo son similares a uno bajo autarquía financiera.

14 Este segundo grupo de parámetros puede ser alternativamente estimado para el período previo a la entrada en vigencia del Tratado de Libre Comercio entre Chile y Estados Unidos (1987 a 2003). Sin embargo, el Test de Wald realizado sobre los datos de comercio de ambos países para la muestra total (1987 a 2007) y para el período previo al tratado revela que ellos no presentan diferencias estadísticamente significativas (ver Anexo A.3). 
TABLA 3

PARAMETROS OBTENIDOS DESDE LA LITERATURA RBC

\begin{tabular}{|ccccl|}
\hline Parámetro & $i=C H$ & & $i=U S$ & \multicolumn{1}{c|}{ Fuente } \\
\hline$\beta$ & & 0,95 & & Bodenstein (2006) \\
$\gamma$ & & 2 & & Backus, Kehoe y Kydland (1992) \\
$\eta$ & & $1 / 3$ & & Betts y Kehoe (2001) \\
$\delta$ & & 0,06 & & Bergoeing et al. (2002) \\
$\psi^{i}$ & $1 / 3$ & & $1 / 3$ & Stockman y Tesar (1995) \\
$\rho_{1}^{i}$ & 0,706 & & 0,633 & Broda, Greenfield y Weinstein (2006) \\
$\rho_{2}^{i}$ & 0,603 & 0,501 & Broda, Greenfield y Weinstein (2006) \\
$\rho_{3}^{i}$ & 0,792 & & 0,791 & Broda, Greenfield y Weinstein (2006) \\
$\theta_{1, i}$ & $1 / 3$ & & $1 / 3$ & Chari, Kehoe y McGrattan (1996) \\
$\theta_{2, i}$ & $1 / 3$ & & $1 / 3$ & Chari, Kehoe y McGrattan (1996) \\
$\theta_{3, i}$ & $1 / 3$ & & $1 / 3$ & Chari, Kehoe y McGrattan (1996) \\
\hline
\end{tabular}

\section{TABLA 4}

DATOS DE COMERCIO CHILE-ESTADOS UNIDOS $(*)$

(Promedio 1987-2007)

\begin{tabular}{|cccc|}
\hline Hecho Estilizado & \multicolumn{3}{c|}{ Sector } \\
\cline { 2 - 4 } & $j=1$ & $j=2$ & $j=3$ \\
\hline$I T_{j}^{C H}$ & 0,173 & 0,292 & 0,067 \\
$X_{j}^{C H} / M_{j}^{C H}$ & 4,415 & 0,445 & 1,180 \\
$c_{j, C H}^{C H} / c_{j, U S}^{U S}$ & 0,016 & 0,002 & 0,002 \\
$y_{j, C H} / y_{j, U S}$ & 0,029 & 0,004 & 0,003 \\
\hline
\end{tabular}

(*): $X_{j}^{C H}=\left(1+\tau_{j, C H}^{U S}\right) c_{j, C H}^{U S} ; M_{j}^{C H}=\left(1+\tau_{j, U S}^{C H}\right) c_{j, U S}^{C H}$.

Las estimaciones de la Tabla 5 muestran que tanto en Chile como en Estados Unidos la preferencia por bienes producidos internamente es mayor que aquella por bienes extranjeros y, en ambos casos, la magnitud estimada difiere entre ambos países. Esta regularidad se repite también a nivel de canastas sectoriales: en Chile los agentes manifiestan una mayor preferencia por bienes del sector manufacturas respecto de Estados Unidos, mientras que en esta última economía las preferencias, relativas a las de Chile, apuntan a bienes del sector primario y construcción y servicios, mayormente. Asimismo, los costos asociados al proceso de exportación muestran una importante 
heterogeneidad a nivel sectorial. De esta forma, la estimación del segundo grupo de parámetros revela que en ambos países existe un sesgo hacia el consumo de bienes domésticos asimétrico. El valor de los ponderadores $a^{C H}$ y $a^{U S}$ estimados se encuentra en línea con Betts y Kehoe (2001), en el sentido de que ellos son proporcionales al producto relativo entre ambos países ${ }^{15}$.

TABLA 5

PARAMETROS ESTIMADOS A PARTIR DE HECHOS ESTILIZADOS

\begin{tabular}{|cccccccc|}
\hline \multirow{2}{*}{ Parámetro } & \multicolumn{3}{c}{$i=C H$} & & & \multicolumn{3}{c|}{$i=U S$} \\
\cline { 2 - 3 } \cline { 6 - 8 } & $j=1$ & $j=2$ & $j=3$ & & $j=1$ & $j=2$ & $j=3$ \\
\hline$a^{i}$ & & 0,005 & & & & 0,995 & \\
$\alpha_{C H}^{i}$ & & 1,143 & & & & 0,638 & \\
$\alpha_{U S}^{i}$ & & 0,884 & & & & 1,286 & \\
$\varsigma_{j}^{i}$ & 1,000 & 1,001 & 1,000 & & 1,256 & 0,743 & 1,158 \\
$\tau_{j, C H}^{i}$ & - & - & - & & 0,301 & 0,301 & 0,299 \\
$\tau_{j, U S}^{i}$ & 0,276 & 0,678 & 0,190 & & - & - & - \\
$A_{j, i}$ & 0,994 & 0,996 & 0,994 & & 0,679 & 0,968 & 0,756 \\
\hline
\end{tabular}

Finalmente, los parámetros de persistencia $\phi_{j, h}$ y la desviación estándar de los errores $\sigma_{\varepsilon_{j, h}}$ fueron estimados de forma que el modelo replique la desviación estándar y autocorrelación del producto de ambos países, del tipo de cambio real, del tipo de cambio real explicado y de las desviaciones desde la Ley de Precio Unico reportadas en la Tabla $2^{16}$. Los resultados se presentan en la Tabla 6.

La versión del modelo con dos países y tres sectores productivos está compuesta por 40 ecuaciones que determinan 40 variables endógenas. Los precios son obtenidos, como se mencionó anteriormente, a partir de las ecuaciones (22) a (24). Finalmente, el modelo fue resuelto mediante la linealización de las 40 ecuaciones en torno al estado estacionario empleando el método de log-linealización ${ }^{17}$.

15 El producto de los sectores primarios, manufacturas y construcción y servicios en Chile alcanzó un promedio de US\$ 40 billones durante el período 1987-2007. Durante igual lapso de tiempo, el producto de Estados Unidos alcanzó un promedio de US\$ 8.430 billones.

16 Las series, expresadas en logaritmos, fueron filtradas mediante el filtro de Hodrick-Prescott empleando un parámetro de penalización $\lambda=100$, tal como lo sugiere esta literatura para datos en frecuencia anual.

17 Para mayores detalles de este método, ver Schmitt-Grohé y Uribe (2004). 


\section{TABLA 6}

ESTIMACION DE PARAMETROS DE PERSISTENCIA Y DESVIACION ESTANDAR DE ERRORES

\begin{tabular}{|cccccc|}
\hline$j$ & \multicolumn{2}{c}{$i=C H$} & & \multicolumn{2}{c|}{$i=U S$} \\
\cline { 2 - 3 } \cline { 5 - 5 } & $\phi_{j, C H}$ & $\sigma_{\varepsilon_{j, C H}}$ & & $\phi_{j, U S}$ & $\sigma_{\varepsilon_{j, U S}}$ \\
\hline 1 & 0,199 & 0,061 & 0,628 & 0,155 \\
2 & 0,490 & 0,226 & & 0,101 & 0,021 \\
3 & 0,289 & 0,054 & & 0,696 & 0,076 \\
\hline
\end{tabular}

\section{RESULTADOS}

En esta sección se comparan los resultados del modelo estimado con los datos del comercio sectorial Chile-Estados Unidos. La comparación emplea la componente cíclica del producto, consumo, tipo de cambio real y tipo de cambio real explicado -todas expresadas en logaritmos-, mientras que la balanza comercial fue tratada como porcentaje del producto. Por último, se realiza una serie de experimentos numéricos como forma de verificar la robustez de los resultados obtenidos.

\subsection{Modelo Base}

En la Tabla 7 se muestran los segundos momentos observados en los datos y los generados por el modelo de las principales variables macroeconómicas estudiadas. Los resultados indican que el modelo es capaz de replicar la mayor volatilidad del tipo de cambio real respecto de variables como el producto, consumo y balanza comercial. Más aún, la variabilidad del consumo agregado predicha por el modelo para Chile y Estados Unidos es prácticamente igual a aquella contenida en los datos. Las principales discrepancias, sin embargo, se encuentran en la desviación estándar y autocorrelación de la balanza comercial y en la autocorrelación del consumo. En ambos casos, el modelo estimado genera una mayor volatilidad y persistencia en las variables de ambos países.

La mayor variabilidad de $R E R$ respecto de $\widehat{R E R}(0,151$ versus 0,090$)$ es un fenómeno que, como se mencionó anteriormente, refleja considerables desviaciones sectoriales desde la Ley de Precio Unico. En este sentido y de acuerdo a los resultados de la Tabla 8, el modelo predice correctamente la matriz de covarianzas asociada al precio relativo de bienes transables $\widetilde{R E R}$ : con un 5\% de significancia, el Test $\mathrm{M}$ de Box no rechaza la hipótesis nula $H_{0}: \hat{\Sigma}_{D}=\hat{\Sigma}_{M}$, donde $\hat{\Sigma}_{D}$ y $\hat{\Sigma}_{M}$ es un estimador para la matriz de covarianzas de los datos y del modelo, respectivamente. 
TABLA 7

DESVIACIONES ESTANDAR Y AUTOCORRELACIONES PRINCIPALES VARIABLES MACROECONOMICAS $(*)$

\begin{tabular}{|cccccc|}
\hline & \multicolumn{2}{c}{ Datos } & & \multicolumn{2}{c|}{ Modelo } \\
\cline { 2 - 3 } \cline { 5 - 6 } & Desv. Est. & Autocorr. & & Desv. Est. & Autocorr. \\
\hline$C^{C H}$ & 0,031 & 0,469 & & 0,028 & 0,887 \\
$C^{U S}$ & 0,016 & 0,795 & & 0,018 & 0,933 \\
$T B^{C H}$ & 0,014 & 0,465 & & 0,025 & 0,947 \\
$T B^{U S}$ & 0,007 & 0,358 & & 0,035 & 0,947 \\
$Y^{C H}$ & 0,070 & 0,559 & 0,070 & 0,559 \\
$Y^{U S}$ & 0,038 & 0,765 & & 0,038 & 0,765 \\
$R E R$ & 0,151 & 0,731 & & 0,151 & 0,731 \\
$\widehat{R E R}$ & 0,090 & 0,153 & & 0,090 & 0,153 \\
\hline
\end{tabular}

$\left.{ }^{*}\right): C^{i}=\sum_{j=1}^{3} c_{j, i}^{i} ; Y^{i}=\sum_{j=1}^{3} y_{j, i} ; T B^{i}=\sum_{j=1}^{3}\left(X_{j}^{i}-M_{j}^{i}\right) / Y^{i}$

TABLA 8

MATRIZ DE VARIANZAS Y COVARIANZAS DESVIACIONES LEY DE PRECIO UNICO SECTORIAL DATOS / (MODELO)

\begin{tabular}{|cccc|}
\hline & $j=1$ & $j=2$ & $j=3$ \\
\hline$j=1$ & 0,077 & & \\
& $(0,077)$ & & \\
$j=2$ & 0,000 & 0,041 & \\
& $(-0,001)$ & $(0,041)$ & 0,027 \\
$j=3$ & 0,009 & 0,030 & $(0,027)$ \\
& $(0,005)$ & $(0,031)$ & \\
\hline
\end{tabular}

Test M de Box: 1,571.

La Tabla 9 reporta las correlaciones entre las principales variables analizadas. A partir de ella se puede apreciar que el modelo presenta ciertas diferencias en las magnitudes estimadas pero es capaz de replicar gran parte de los signos de comovimiento, en especial el carácter contracíclico de la balanza comercial ${ }^{18}$. La diferencia más notable entre el modelo y los datos corresponde al signo de la correlación entre

18 Ver, por ejemplo, Backus, Kehoe y Kydland (1994). 
el ratio de consumo y el tipo de cambio real, diferencia conocida en el campo de la macroeconomía internacional como el puzzle Backus-Smith ${ }^{19}$. En el contexto del modelo empleado, la literatura más reciente relacionada a este puzzle sugiere la consideración de costos de transacción "levemente mayores que aquellos tradicionalmente utilizados" para resolver esta anomalía. Observando los costos de transacción estimados en la Tabla 5 y considerando como referencia un valor igual a 0,3 -valor estimado por Obstfeld y Rogoff (2000) que es ampliamente utilizado en la literatura de tipo de cambio real y que puede ser considerado como "tradicional"- la incapacidad del modelo para resolver el puzzle Backus-Smith se explicaría por los bajos valores estimados para los costos de transacción y la existencia de autarquía financiera ${ }^{20}$.

\section{TABLA 9}

CORRELACIONES CRUZADAS PRINCIPALES VARIABLES MACROECONOMICAS DATOS / (MODELO)

\begin{tabular}{|ccccccc|}
\hline & $\left(C^{C H} / C^{U S}\right)$ & $T B^{C H}$ & $T B^{U S}$ & $Y^{C H}$ & $Y^{U S}$ & RER \\
\hline \multirow{2}{*}{$T B^{C H}$} & $-0,810$ & & & & \\
& $(0,970)$ & & & & \\
$T B^{U S}$ & $-0,760$ & $-0,955$ & & & & \\
& $(-0,971)$ & $(-0,998)$ & & & & \\
$Y^{C H}$ & 0,874 & $-0,838$ & $-0,780$ & & & \\
& $(0,612)$ & $(-0,530)$ & $(-0,531)$ & & & \\
$Y^{U S}$ & $-0,206$ & 0,403 & $-0,354$ & 0,183 & & \\
& $(-0,676)$ & $(0,573)$ & $(-0,574)$ & $(0,067)$ & & \\
$R E R$ & $-0,879$ & 0,624 & $-0,520$ & $-0,725$ & 0,165 & \\
& $(0,632)$ & $(0,621)$ & $(-0,621)$ & $(-0,267)$ & $(0,417)$ & \\
$\widehat{R E R}$ & $-0,037$ & 0,172 & $-0,281$ & $-0,111$ & 0,449 & 0,287 \\
& $(-0,013)$ & $(0,111)$ & $(-0,111)$ & $(-0,325)$ & $(0,355)$ & $(0,586)$ \\
\hline
\end{tabular}

De este modo, los resultados obtenidos indican que la versión de un sencillo modelo con dos países y tres sectores productivos es capaz de replicar la mayor volatilidad del tipo de cambio real bilateral Chile-Estados Unidos respecto de los principales agregados macroeconómicos observados en los datos, lo cual se explicaría por la

19 Este puzzle, documentado inicialmente por Backus y Smith (1993), postula la existencia de una correlación empírica nula -o incluso negativa- entre el ratio de consumo y el tipo de cambio real. No obstante lo anterior, Obstfeld y Rogoff (2000) indican que la existencia de este puzzle depende de la especificación del modelo empleado, en especial de factores como el grado de completitud de los mercados financieros internacionales y la forma exacta de la función de utilidad.

20 Ver, por ejemplo, Obstfeld y Rogoff (2000) y Corsetti, Dedola y Leduc (2005). 
existencia de costos de transacción y el sesgo asimétrico hacia el consumo sectorial de bienes domésticos provocado por los distintos grados de sustitución imperfecta entre bienes locales y extranjeros. Los primeros explicarían los desvíos sectoriales desde la Ley de Precio Unico, mientras que los segundos inducirían una baja correlación entre el consumo de ambos países, lo que finalmente se traduciría en las mayores fluctuaciones del tipo de cambio real ${ }^{21}$.

\subsection{Análisis de Sensibilidad}

Existen tres aspectos adicionales que se desprenden de los resultados anteriores y que ayudan a precisar las conclusiones acerca de las fluctuaciones del tipo de cambio real generadas por el modelo. El primer aspecto apunta al grado de asimetría en el sesgo hacia el consumo de bienes domésticos (home bias effect). El segundo se relaciona con el grado de impaciencia de los agentes como señaló Bodenstein (2006), mientras que el tercero se basa en los resultados de Chari, Kehoe y McGrattan (2001) referentes al valor de la aversión relativa al riesgo.

Para abordar el primer aspecto, el modelo estimado (modelo base) es contrastado con dos escenarios alternativos: (i) ausencia del efecto home bias, donde $\tau_{j, h}^{i}=0,3, \alpha_{h}^{h}=1$ y $\varsigma_{j}^{i}=1$; y (ii) un efecto home bias simétrico, donde $\tau_{j, h}^{i}=0,3, \alpha_{h}^{h}=1,143, \alpha_{h}^{i}=0,884 \mathrm{y}$ $\varsigma_{j}^{i}=1^{22}$. Los resultados de la Tabla 10 muestran que en el primer escenario el modelo genera una volatilidad del tipo de cambio real y desviaciones sectoriales desde la Ley de Precio Unico inferiores a las observadas en los datos y en el modelo base.

Consistente con la teoría tradicional, el tipo de cambio real explicado ante la ausencia del efecto home bias es casi tan volátil como RER y representa poco menos del $90 \%$ de sus fluctuaciones. Bajo un escenario con home bias simétrico, los resultados del modelo tienden a mejorar sólo a nivel sectorial: las desviaciones desde la Ley de Precio Unico son mayores en comparación con el primer caso, pero se mantienen aún por debajo de las observadas en los datos; y si bien la volatilidad de $\widehat{R E R}$ tiende a explicar una menor porción de las fluctuaciones del tipo de cambio real (cerca del $81 \%$ ), esta última se mantiene prácticamente inalterada. Así, los resultados de la Tabla 10 indican que la mayor volatilidad del tipo de cambio real predicha por el modelo, respecto de variables como el consumo y el producto, se explicaría por la existencia de preferencias en el consumo de bienes domésticos y extranjeros distintas entre agentes, es decir, por un efecto home bias asimétrico generado de forma endógena por el modelo.

21 La correlación entre el consumo de Chile y Estados Unidos predicha por el modelo es igual a 0,117.

22 Los valores elegidos para $\alpha_{h}^{h}$ y $\alpha_{h}^{i}$ corresponden a aquellos estimados para Chile. La consideración alternativa de los valores estimados para Estados Unidos no altera los resultados finales. 


\section{TABLA 10}

GRADO DE ASIMETRIA EFECTO HOME BIAS
ESPECIFICACIONES ALTERNATIVAS

\begin{tabular}{|lcccc|}
\hline & $\begin{array}{c}\text { Ausencia } \\
\text { Home Bias }\end{array}$ & $\begin{array}{c}\text { Home Bias } \\
\text { Simétrico }\end{array}$ & $\begin{array}{c}\text { Home Bias } \\
\text { Asimétrico }(*)\end{array}$ & Datos \\
\hline$\sigma_{R E R}$ & 0,125 & 0,123 & 0,151 & 0,151 \\
$\sigma_{\widehat{R E R}}$ & 0,112 & 0,100 & 0,090 & 0,090 \\
$D_{1}^{L P U}$ & 0,215 & 0,240 & 0,277 & 0,277 \\
$D_{2}^{L P U}$ & 0,167 & 0,176 & 0,203 & 0,203 \\
$D_{3}^{L P U}$ & 0,103 & 0,130 & 0,166 & 0,166 \\
\hline
\end{tabular}

(*): Modelo base.

Para indagar acerca de los efectos sobre las fluctuaciones del tipo de cambio real ocasionados por distintos grados de impaciencia de los agentes, el modelo es estimado para valores del factor de descuento intertemporal $\beta$ usualmente utilizados en la literatura de ciclos reales (Tabla 11).

\section{TABLA 11}

GRADO DE IMPACIENCIA DE LOS AGENTES

ESPECIFICACIONES ALTERNATIVAS

\begin{tabular}{|lcccc|}
\hline & $\beta=0,90$ & $\beta=0,95\left(^{*}\right)$ & $\beta=0,97$ & $\beta=0,99$ \\
\hline$\sigma_{\text {RER }}$ & 0,153 & 0,151 & 0,151 & 0,150 \\
$\sigma_{\widehat{R E R}}$ & 0,089 & 0,090 & 0,090 & 0,092 \\
$D_{1}^{L P U}$ & 0,306 & 0,277 & 0,261 & 0,239 \\
$D_{2}^{L P U}$ & 0,226 & 0,203 & 0,190 & 0,174 \\
$D_{3}^{L P U}$ & 0,184 & 0,166 & 0,155 & 0,141 \\
\hline
\end{tabular}

(*): Modelo base.

Consistente con Bodenstein (2006), a medida que el valor de $\beta$ disminuye el modelo tiende a generar una mayor volatilidad en el tipo de cambio real y en el precio relativo sectorial de bienes transables. El mecanismo implícito que explicaría este resultado indica que bajo autarquía financiera y agentes suficientemente impacientes, 
la correlación entre el consumo de ambos países es baja, lo que se traduciría finalmente en mayores fluctuaciones de RER. No obstante lo anterior, las ganancias en términos de volatilidad del tipo de cambio real al incluir menores valores de $\beta$ en el modelo son despreciables: la diferencia en $\sigma_{R E R}$ al considerar un factor de descuento intertemporal igual a 0,90 respecto del escenario base $(\beta=0,95)$ es de sólo 0,002 , es decir, un aumento en la volatilidad de RER de poco más del $1 \%$.

Con respecto al último aspecto mencionado, Chari, Kehoe y McGrattan (2001) postulan que si la aversión relativa al riesgo de los agentes $\gamma$ es elevada (enfoque de aversión al riesgo), entonces la variabilidad del consumo relativo $\left(\mathrm{C}^{\mathrm{CH}} / \mathrm{C}^{U S}\right)$ observada en los datos podría generar, en el equilibrio, grandes movimientos del tipo de cambio real. De esta forma, el modelo es estimado para $\gamma$ comprendido en el intervalo $[1,6]$. Los resultados se muestran en la Tabla 12.

TABLA 12

GRADO DE AVERSION RELATIVA AL RIESGO ESPECIFICACIONES ALTERNATIVAS

\begin{tabular}{|lllllll|}
\hline & $\gamma=1$ & $\gamma=2\left(^{*}\right)$ & $\gamma=3$ & $\gamma=4$ & $\gamma=5$ & $\gamma=6$ \\
\hline$\sigma_{R E R}$ & 0,149 & 0,151 & 0,152 & 0,153 & 0,154 & 0,155 \\
$\sigma_{\widehat{R E R}}$ & 0,090 & 0,090 & 0,090 & 0,090 & 0,090 & 0,090 \\
$D_{1}^{L P U}$ & 0,275 & 0,277 & 0,279 & 0,281 & 0,282 & 0,282 \\
$D_{2}^{L P U}$ & 0,203 & 0,203 & 0,203 & 0,204 & 0,204 & 0,204 \\
$D_{3}^{L P U}$ & 0,162 & 0,166 & 0,168 & 0,170 & 0,172 & 0,172 \\
\hline
\end{tabular}

(*): Modelo base.

A partir de la tabla anterior es posible confirmar la relación positiva entre $\sigma_{R E R}$ y $\gamma$ planteada por los autores. Considerando $\gamma=6$, la volatilidad del tipo de cambio real aumenta respecto del modelo base como consecuencia del mayor incremento en los desvíos desde la Ley de Precio Unico del sector primario $(1,8 \%)$ y construcción y servicios $(3,6 \%)$. La volatilidad del tipo de cambio real explicado se mantiene prácticamente inalterada, hecho que podría deberse al aumento marginal de $D_{2}^{L P U}$ $(0,5 \%)$ y los mayores incrementos en la volatilidad del sector no transable cuando la aversión relativa al riesgo aumenta de 2 a 6 . Sin embargo, y al igual que en el caso anterior, las ganancias en términos de mayores fluctuaciones de $R E R$ son pequeñas. 


\section{CONCLUSIONES}

La teoría tradicional asociada a la determinación del tipo de cambio real postula que las fluctuaciones de dicha variable son consecuencia de movimientos en el precio relativo de bienes transables a no transables. Sin embargo, diversos estudios empíricos han mostrado que la volatilidad del tipo de cambio real es mayor que aquella observada en variables como el consumo y el producto, además de presentar desvíos considerables desde la Ley de Precio Unico. En este sentido y basado en el trabajo de Cole y Obstfeld (1991); Betts y Kehoe (2001); y Heathcote y Perri (2002), el presente artículo desarrolla un modelo dinámico de equilibrio general estocástico multipaís y multisector bajo autarquía financiera e inexistencia del dinero para explicar tales fluctuaciones, el cual es estimado para los sectores primario, manufacturas y construcción y servicios asociados al comercio internacional entre Chile y Estados Unidos.

Los resultados obtenidos indican que el modelo estimado es capaz de predecir las mayores fluctuaciones del tipo de cambio real bilateral y los desvíos sectoriales desde la Ley de Precio Unico, los cuales se explicarían por la existencia de costos de transacción asociados al comercio internacional y distintos grados de sustitución imperfecta entre el consumo de bienes domésticos y extranjeros que son consistentes con los datos. Intuitivamente, los costos de transacción estimados -bastante similares a aquellos reportados por Obstfeld y Rogoff (2000)- explicarían los desvíos desde la Ley de Precio Unico observados en los sectores primario, manufacturas y construcción y servicios, mientras que los distintos grados de sustitución entre bienes nacionales e importados inducen un efecto home bias asimétrico que sería responsable de la baja correlación entre el consumo de Chile y Estados Unidos, y finalmente, de la mayor volatilidad del tipo de cambio real bilateral; resultados que son confirmados por el análisis de sensibilidad realizado.

Finalmente, el análisis de correlaciones entre las principales variables macroeconómicas estudiadas revela que el modelo es capaz de replicar gran parte de los signos de comovimiento entre ellas, en especial, el carácter contracíclico de la balanza comercial de Chile y Estados Unidos. La diferencia más notable entre el modelo y los datos, sin embargo, corresponde al signo de la correlación entre el ratio de consumo y el tipo de cambio real (puzzle de Backus-Smith), el cual, en el contexto del modelo empleado, se debería a que los costos de transacción estimados son menores o iguales respecto del valor tradicionalmente utilizado en esta literatura.

\section{REFERENCIAS}

ARVANTIS, A.V. y A. MIKKOLA (1996). "Asset-Market Structure and International Trade Dynamics", American Economic Paper and Proceedings 86, pp. 67-70.

BACKUS, D.K., P.J. KEHOE y F.E. KYDLAND (1992). "International Real Business Cycles", Journal of Political Economy 100, pp. 745-775. 
BACKUS, D.K., P.J. KEHOE y F.E. KYDLAND (1994). "Dynamics of the Trade Balance and the Terms of Trade: The J-Curve", American Economic Review 84, pp. 84-103.

BACKUS, D.K. y G.W. SMITH (1993). "Consumption and Real Exchange Rates in Dynamic Economies with Non-Traded Goods", Journal of International Economics 35, pp. 297-316.

BAXTER, M. y M.J. CRUCINI (1995). "Business Cycles and the Asset Structure of Foreign Trade", International Economic Review 36 (4), pp. 821-854.

BERGOEING, R., P.J. KEHOE, T.J. KEHOE y R. SOTO (2002). "A Decade Lost and Found: Mexico and Chile in the 1980s", NBER Working Paper Nr. 8520, National Bureau of Economic Research.

BETTS, C.M. y M.B. DEVEREUX (2000). "Exchange Rate Dynamics in a Model of Pricing-to-Market", Journal of International Economics 50, pp. 215-244.

BETTS, C.M. y T.J. KEHOE (2001). “Tradability of Goods and Real Exchange Rate Fluctuations”, Federal Reserve Bank of Minneapolis y University of Southern California.

BETTS, C.M. y T.J. KEHOE (2005). "U.S. Exchange Rate Fluctuations and Relative Price Fluctuations", Staff Report Nr. 334, Federal Reserve Bank of Minneapolis.

BETTS, C.M. y T.J. KEHOE (2008). "Real Exchange Rate Movements and the Relative Price of NonTraded Goods", Staff Report Nr. 415, Federal Reserve Bank of Minneapolis.

BODENSTEIN, M. (2006). "International Asset Markets and Real Exchange Rate Volatility", International Finance Discussion Paper Nr. 884, Board of Governors of the Federal Reserve System.

BRODA, C., J. GREENFIELD y D.E. WEINSTEIN (2006). "From Groundnuts to Globalization: A Structural Estimate of Trade and Growth", NBER Working Paper Nr. 12512, National Bureau of Economic Research.

CHARI, V., P. J. KEHOE y E. McGRATTAN (1996). "The Poverty of Nations: A Quantitative Exploration", Staff Report Nr. 204, Federal Reserve Bank of Minneapolis.

CHARI, V., P.J. KEHOE y E. McGRATTAN (2001). "Can Sticky Price Models Generate Volatile and Persistent Real Exchange Rates?”, Staff Report Nr. 277, Federal Reserve Bank of Minneapolis.

COLE, H.L. y M. OBSTFELD (1991). "Commodity Trade and International Risk Sharing: How Much Do Financial Markets Matter?”, Journal of Monetary Economics 28, pp. 3-24.

CORSETTI, G., L. DEDOLA y S. LEDUC (2004). "International Risk Sharing and the Transmission of Productivity Shocks", Discussion Paper Nr. 4746, Centre for Economic Policy Research.

CORSETTI, G., L. DEDOLA y S. LEDUC (2005). "DSGE Models of High Exchange-Rate Volatility and Low Pass-Through", International Finance Discussion Paper Nr. 845, Board of Governors of the Federal Reserve System.

ENGEL, C. (1993). "Real Exchange Rates and Relative Prices: An Empirical Investigation", Journal of Monetary Economics 32, pp. 35-50.

ENGEL, C. (1999). "Accounting for U.S. Real Exchange Rate Changes", Journal of Political Economy 107 , pp. 507-538.

ENGEL, C. y J.H. ROGERS (1996). "How Wide is the Border?", American Economic Review 86, pp. 1112-1125.

GIOVANNINI, A. (1988). "Exchange Rates and Traded Goods Prices", Journal of International Economics 24, pp. 45-68.

HEATHCOTE, J. y F. PERRI (2002). "Financial Autarky and International Business Cycles", Journal of Monetary Economics 49, pp. 601-622.

HELLIWELL, J.F. (1996). "Do National Borders Matter for Quebec Trade?", Canadian Journal of Economics 29, pp. 507-522.

ISARD, P. (1977). "How Far Can We Push the Law of One Price?", American Economic Review 67, pp. 942-948.

KING, R.G., C.I. PLOSSER y S.T. REBELO (1988). "Production, Growth and Business Cycles: I. The Basic Neoclassical Model", Journal of Monetary Economics 21, pp. 195-232.

KNETTER, M.M. (1997). "International Comparisons of Price-to-Market Behavior", American Economic Review 83, pp. 473-486.

KOLLMAN, R. (1996). "Incomplete Asset Markets and the Cross-Country Correlation Puzzle", Journal of Economic Dynamics and Control 20, pp. 945-961. 
LAPHAM, B.J. (1995). "A Dynamic General Equilibrium Analysis of Deviations from the Laws of One Price", Journal of Economic Dynamics and Control 19, pp. 1355-1389.

LIPSEY, R.E. e I.B. KRAVIS (1978). "Price Behavior in the Light of Balance of Payments Theories", Journal of International Economics 8, pp. 193-246.

McCALLUM, J. (1995). "National Borders Matter: Canada-US Regional Trade Patterns", American Economic Review 85 (3), pp. 615-623.

OBSTFELD, M. y K. ROGOFF (2000). "The Six Major Puzzles in International Macroeconomics: Is There a Common Cause?”, NBER Working Paper Nr. 7777, National Bureau of Economic Research.

SCHMITT-GROHE, S. y M. URIBE (2004). "Solving Dynamic General Equilibrium Models Using a Second-Order Approximation to the Policy Function”, Journal of Economic Dynamics and Control 28, pp. 755-775.

STOCKMAN, A.C. y L.L. TESAR (1995). "Tastes and Technology in a Two-Country Model of Business Cycle: Explaining International Comovements", American Economic Review 85, pp. 168-185. 


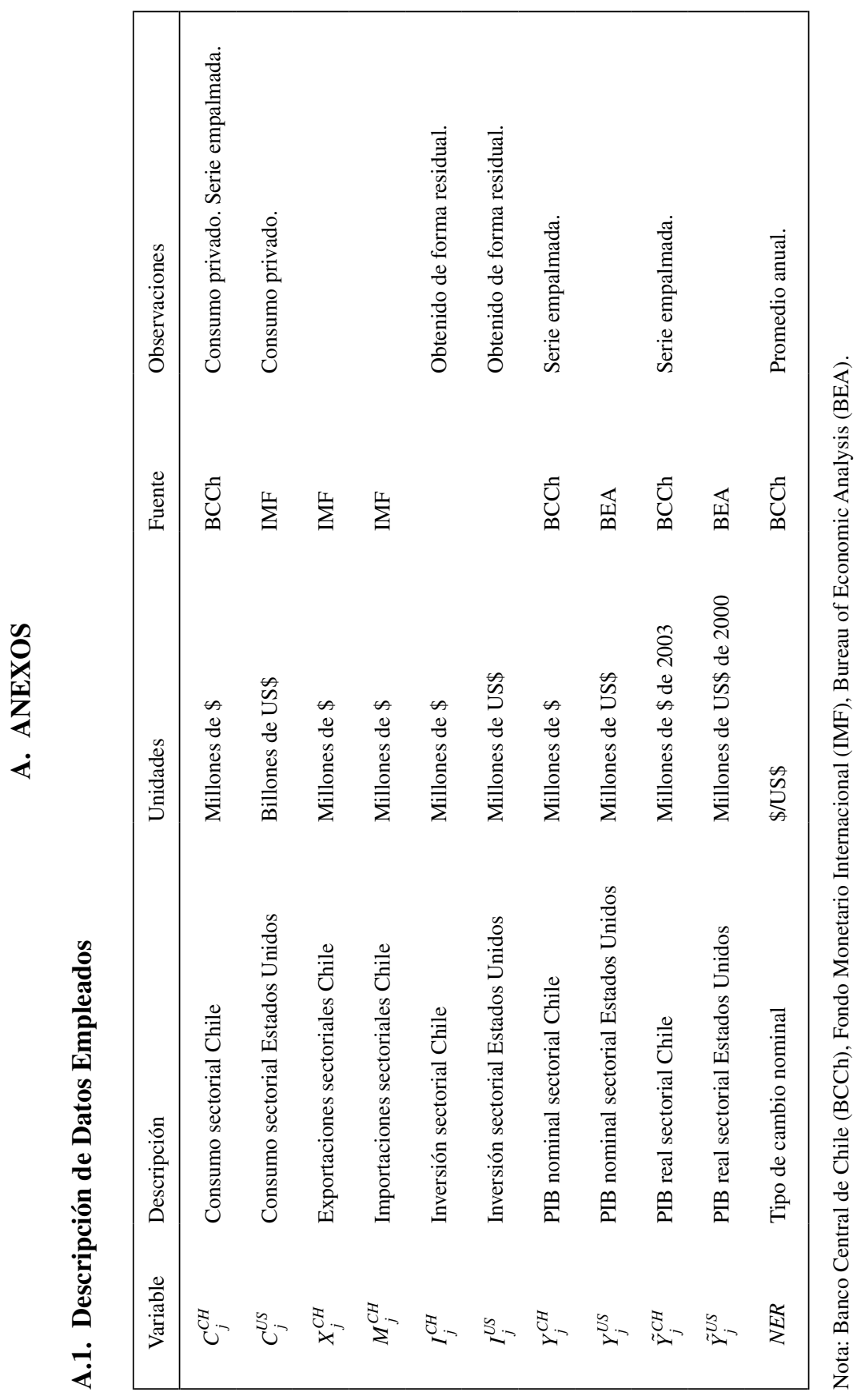




\section{A.2. Equivalencia entre el Equilibrio Descentralizado y Centralizado}

En este Anexo se resumen las condiciones de equilibrio asociadas al modelo descentralizado y al del planificador mundial (centralizado), y finalmente se deriva la equivalencia entre ambos.

\section{A.2.1. Condiciones de Equilibrio del Modelo Descentralizado}

Las condiciones de primer orden del modelo para $i, h \in I, i \neq h$, y $j, q \in J$, $j \neq q$, se encuentran dadas por:

$$
\begin{gathered}
\frac{p_{q, h, t}}{p_{j, h, t}}=\frac{\partial u^{h}\left(c_{t}^{h}, 1-l_{t}^{h}\right)}{\partial c_{q, h, t}^{h}} \cdot\left(\frac{\partial u^{h}\left(c_{t}^{h}, 1-l_{t}^{h}\right)}{\partial c_{j, h, t}^{h}}\right)^{-1} \\
\frac{1}{1+\tau_{j, i}^{h}}=\frac{p_{j, i, t}}{p_{j, h, t}} \cdot \frac{\partial u^{h}\left(c_{t}^{h}, 1-l_{t}^{h}\right)}{\partial c_{j, h, t}^{h}} \cdot\left(\frac{\partial u^{h}\left(c_{t}^{h}, 1-l_{t}^{h}\right)}{\partial c_{j, i, t}^{h}}\right)^{-1} \\
\frac{w_{t}^{h}}{p_{j, h, t}}=\frac{\partial u^{h}\left(c_{t}^{h}, 1-l_{t}^{h}\right)}{\partial l_{t}^{h}} \cdot\left(\frac{\partial u^{h}\left(c_{t}^{h}, 1-l_{t}^{h}\right)}{\partial c_{j, h, t}^{h}}\right)^{-1} \\
{\left[\begin{array}{c}
\frac{p_{j, h, t}}{\beta} \cdot \frac{\partial u^{h}\left(c_{t+1}^{h}, 1-l_{t+1}^{h}\right)}{\partial c_{j, h, t+1}^{h}} \cdot\left(\frac{\partial u^{h}\left(c_{t}^{h}, 1-l_{t}^{h}\right)}{\partial c_{j, h, t}^{h}}\right)^{-1} \\
\frac{\left.p_{j, h, t+1}^{h}+1-\delta\right)}{r_{t+1}}
\end{array}\right.} \\
\frac{w_{t}^{h}}{p_{j, h, t}}=\frac{\partial f\left(k_{j, t}^{h}, l_{j, t}^{h}, z_{j, h, t}\right)}{\partial l_{j, t}^{h}} \\
{\left[\begin{array}{l}
\partial f\left(k_{j, t}^{h}, l_{j, t}^{h}, z_{j, h, t}\right) \\
\partial k_{j, t}^{h}
\end{array}\right.}
\end{gathered}
$$




\section{A.2.2. Condiciones de Equilibrio del Modelo Centralizado}

De forma análoga, las condiciones de primer orden del modelo del planificador mundial para $i, h \in I, i \neq h$, y $j, q \in J, j \neq q$, se encuentran dadas por:

$$
\begin{gathered}
\frac{1}{1+\tau_{j, i}^{h}}=\frac{a^{i}}{a^{h}} \cdot \frac{\partial u^{i}\left(c_{t}^{i}, 1-l_{t}^{i}\right)}{\partial c_{j, i, t}^{i}} \cdot\left(\frac{\partial u^{h}\left(c_{t}^{h}, 1-l_{t}^{h}\right)}{\partial c_{j, i, t}^{h}}\right)^{-1} \\
\frac{\partial f\left(k_{j, t}^{h}, l_{j, t}^{h}, z_{j, h, t}\right)}{\partial l_{j, t}^{h}}=\frac{\partial u^{h}\left(c_{t}^{h}, 1-l_{t}^{h}\right)}{\partial l_{j, t}^{h}} \cdot\left(\frac{\partial u^{h}\left(c_{t}^{h}, 1-l_{t}^{h}\right)}{\partial c_{j, h, t}^{h}}\right)^{-1} \\
\frac{1}{\beta}=E_{t}\left[\left(\frac{\partial f\left(k_{j, t+1}^{h}, l_{j, t+1}^{h}, z_{j, h, t+1}\right)}{\partial k_{j, t+1}^{h}}+1-\delta\right) \cdot \frac{\partial u^{h}\left(c_{t+1}^{h}, 1-l_{t+1}^{h}\right)}{\partial c_{j, h, t+1}^{h}} \cdot\left(\frac{\partial u^{h}\left(c_{t}^{h}, 1-l_{t}^{h}\right)}{\partial c_{j, h, t}^{h}}\right)^{-1}\right]
\end{gathered}
$$

en conjunto con los precios dados por las ecuaciones (22) a (24).

\section{A.2.3. Equivalencia}

Consideremos el precio de los bienes $j$ y $q(j \neq q)$ producidos en el país $h$. A partir de la ecuación (22), el precio relativo entre ambos bienes, $\left(p_{q, h, t} / p_{j, h, t}\right)$, se encuentra dado por:

$$
\frac{p_{q, h, t}}{p_{j, h, t}}=\frac{\partial u^{h}\left(c_{t}^{h}, 1-l_{t}^{h}\right)}{\partial c_{q, h, t}^{h}} \cdot\left(\frac{\partial u^{h}\left(c_{t}^{h}, 1-l_{t}^{h}\right)}{\partial c_{j, h, t}^{h}}\right)^{-1}, \quad h \in I, \quad j, q \in J
$$

Notamos que la ecuación (A.10) es idéntica a la ecuación (A.1) e indica que el precio del bien $q$ relativo al precio del bien $j$ es simplemente la razón entre la utilidad marginal asociada al consumo de ambos bienes.

Consideremos ahora el precio del bien $j$ producido por los países $i$ y $h(i \neq h)$. Empleando nuevamente la ecuación (22), el precio relativo entre ambos países, $\left(p_{j, i, t}\right.$ $\left./ p_{j, h, t}\right)$, está dado por:

$$
\frac{p_{j, i, t}}{p_{j, h, t}}=\frac{a^{i}}{a^{h}} \cdot \frac{\partial u^{i}\left(c_{t}^{i}, 1-l_{t}^{i}\right)}{\partial c_{j, i, t}^{i}} \cdot\left(\frac{\partial u^{h}\left(c_{t}^{h}, 1-l_{t}^{h}\right)}{\partial c_{j, i, t}^{h}}\right)^{-1}, \quad i, h \in I, \quad j \in J
$$


Reemplazando la ecuación (A.11) en (A.7) y luego de reordenar términos, se obtiene:

$$
\frac{1}{1+\tau_{j, i}^{h}}=\frac{p_{j, i, t}}{p_{j, h, t}} \cdot \frac{\partial u^{h}\left(c_{t}^{h}, 1-l_{t}^{h}\right)}{\partial c_{j, h, t}^{h}} \cdot\left(\frac{\partial u^{h}\left(c_{t}^{h}, 1-l_{t}^{h}\right)}{\partial c_{j, i, t}^{h}}\right)^{-1}, \quad i, h \in I, \quad j \in J
$$

La ecuación anterior indica que la razón entre la utilidad marginal asociada al consumo del bien doméstico $\left(c_{j, h, t}^{h}\right)$ y la utilidad marginal asociada al consumo del bien importado $\left(c_{j, i, t}^{h}\right)$, expresada en términos del precio relativo $\left(p_{j, i, t} / p_{j, h, t}\right)$, es igual al inverso del costo de transacción $\tau_{j, i}^{h}$; expresión idéntica a la postulada por la ecuación (A.2).

Por otra parte, las condiciones (A.3) y (A.5) derivadas del modelo descentralizado corresponden a la oferta y demanda por trabajo, respectivamente. La primera postula que el salario real de equilibrio en términos del precio del bien doméstico $j$, $\left(w_{t}^{h} / p_{j, h, t}\right)$, es igual a la razón entre la utilidad marginal del trabajo total $\left(l_{t}^{h}\right)$ y la utilidad marginal del consumo del bien doméstico $j\left(c_{j, h, t}^{h}\right)$. La segunda condición implica que $\left(w_{t}^{h} / p_{j, h, t}\right)$ es igual a la productividad marginal del trabajo empleado en el sector productivo $j\left(l_{j, t}^{h}\right)$. Igualando la demanda con la oferta por trabajo se tiene que:

$$
\frac{\partial f\left(k_{j, t}^{h}, l_{j, t}^{h}, z_{j, h, t}\right)}{\partial l_{j, t}^{h}}=\frac{\partial u^{h}\left(c_{t}^{h}, 1-l_{t}^{h}\right)}{\partial l_{t}^{h}} \cdot\left(\frac{\partial u^{h}\left(c_{t}^{h}, 1-l_{t}^{h}\right)}{\partial c_{j, h, t}^{h}}\right)^{-1}, \quad h \in I, \quad j \in J
$$

La utilidad marginal del trabajo empleado en el sector $j,\left(\partial u^{h}(\cdot) / \partial l_{j, t}^{h}\right)$, puede ser reescrita de la siguiente forma:

$$
\frac{\partial u^{h}\left(c_{t}^{h}, 1-l_{t}^{h}\right)}{\partial l_{j, t}^{h}}=\frac{\partial u^{h}\left(c_{t}^{h}, 1-l_{t}^{h}\right)}{\partial l_{t}^{h}} \cdot \frac{\partial l_{t}^{h}}{\partial l_{j, t}^{h}}, \quad h \in I, \quad j \in J
$$

Debido a que el trabajo es inmóvil internacionalmente, $l_{t}^{h}=\sum_{j=1}^{J} l_{j, t}^{h}, h \in I, \mathrm{y}$ por lo tanto, $\left(\partial l_{t}^{h} / \partial l_{j, t}^{h}\right)=1, h \in I, j \in J$. Así, la ecuación (A.14) se reduce a:

$$
\frac{\partial u^{h}\left(c_{t}^{h}, 1-l_{t}^{h}\right)}{\partial l_{j, t}^{h}}=\frac{\partial u^{h}\left(c_{t}^{h}, 1-l_{t}^{h}\right)}{\partial l_{t}^{h}}, \quad h \in I, \quad j \in J
$$


Reemplazando (A.15) en (A.8):

$$
\frac{\partial f\left(k_{j, t}^{h}, l_{j, t}^{h}, z_{j, h, t}\right)}{\partial l_{j, t}^{h}}=\frac{\partial u^{h}\left(c_{t}^{h}, 1-l_{t}^{h}\right)}{\partial l_{t}^{h}} \cdot\left(\frac{\partial u^{h}\left(c_{t}^{h}, 1-l_{t}^{h}\right)}{\partial c_{j, h, t}^{h}}\right)^{-1}, \quad h \in I, \quad j \in J
$$

que es idéntica a la igualdad entre demanda y oferta de trabajo derivada a partir de las condiciones de primer orden del modelo descentralizado (ecuación (A.13)).

Finalmente, para demostrar la equivalencia entre las ecuaciones de Euler del modelo descentralizado y aquellas obtenidas desde el modelo del planificador mundial, el crecimiento bruto del precio del bien doméstico $j,\left(p_{j, h, t+1} / p_{j, h, t}\right)$, puede ser obtenido a partir de la ecuación (22) y se encuentra dado por:

$$
\frac{p_{j, h, t+1}}{p_{j, h, t}}=\frac{\partial u^{h}\left(c_{t+1}^{h}, 1-l_{t+1}^{h}\right)}{\partial c_{j, h, t+1}^{h}} \cdot\left(\frac{\partial u^{h}\left(c_{t}^{h}, 1-l_{t}^{h}\right)}{\partial c_{j, h, t}^{h}}\right)^{-1}, \quad h \in I, \quad j \in J
$$

Reemplazando (A.17) y la expresión para el interés dado por la ecuación (24) en la ecuación de Euler centralizada (A.9), y luego de reordenar términos, se obtiene:

$$
\begin{aligned}
& \frac{1}{\beta}=E_{t}\left[\left(r_{t+1}^{h}+1-\delta\right) \cdot \frac{p_{j, h, t}}{p_{j, h, t+1}} \cdot \frac{\partial u^{h}\left(c_{t+1}^{h}, 1-l_{t+1}^{h}\right)}{\partial c_{j, h, t+1}^{h}}\right. \\
& \left.\left(\frac{\partial u^{h}\left(c_{t}^{h}, 1-l_{t}^{h}\right)}{\partial c_{j, h, t}^{h}}\right)^{-1}\right]
\end{aligned}
$$

Notamos que la ecuación (A.18) es igual a la ecuación (A.4). De esta forma, las condiciones de primer orden obtenidas a partir del modelo del planificador mundial son equivalentes a aquellas obtenidas a partir del modelo descentralizado. 


\section{A.2.4. Tipo de Cambio Real y Tipo de Cambio Real Explicado bajo el Modelo Centralizado}

Bajo inexistencia del dinero, la ecuación (1) indica que el tipo de cambio real entre los países $i$ y $h, R E R_{t}$, corresponde al precio agregado del país $i\left(P_{t}^{i}\right)$ relativo al precio agregado del país $h\left(P_{t}^{h}\right)$ :

$$
R E R_{t}=\frac{P_{t}^{i}}{P_{t}^{h}}, \quad i, h \in I
$$

Si la Ley de Precio Unico se cumple en cada período $t$, entonces el tipo de cambio real explicado entre los países $i$ y $h, \widehat{R E R}_{t}$, puede ser obtenido desde la ecuación (3) como:

$$
\widehat{R E R}_{t}=\frac{P T_{t}^{h}}{P T_{t}^{i}} \cdot \frac{P_{t}^{i}}{P_{t}^{h}}, \quad i, h \in I
$$

donde $P T_{t}^{i}$ y $P T_{t}^{h}$ corresponden al precio agregado de bienes transables del país $i$ y del país $h$, respectivamente.

Sea $J_{1}$ el conjunto de todos los bienes transables y $J_{2}$ el conjunto de todos los bienes no transables de la economía mundial, tales que $J_{1} \cup J_{2}=J$. A partir de esta definición, los índices de precios (6) y (7) y los precios definidos por (22), $R E R_{t}$ y $\widehat{R E R}_{t}$ se encuentran dados por:

$$
R E R_{t}=\frac{a^{i}}{a^{i}} \cdot\left[\frac{\sum_{j \in J}\left(\frac{\partial u^{i}\left(c_{t}^{i}, 1-l_{t}^{i}\right)}{\partial c_{j, i, t}^{i}} \cdot y_{j, i, t}\right)}{\sum_{j \in J} y_{j, i, t}}\right] \cdot\left[\frac{\sum_{j \in J}\left(\frac{\partial u^{h}\left(c_{t}^{h}, 1-l_{t}^{h}\right)}{\partial c_{j, h, t}^{i}} \cdot y_{j, h, t}\right)}{\sum_{j \in J} y_{j, h, t}}\right]^{-1}
$$




$$
\left.\begin{array}{rl}
\widehat{R E R}_{t}= & {\left[\frac{\sum_{j \in J}\left(\frac{\partial u^{i}\left(c_{t}^{i}, 1-l_{t}^{i}\right)}{\partial c_{j, i, t}^{i}} \cdot y_{j, i, t}\right)}{\sum_{j \in J} y_{j, i, t}}\right] \cdot\left[\frac{\sum_{j \in J_{1}}\left(\frac{\partial u^{h}\left(c_{t}^{h}, 1-l_{t}^{h}\right)}{\partial c_{j, h, t}^{i}} y_{j, h, t}\right)}{\sum_{j \in J_{1}} y_{j, h, t}}\right]} \\
& \left.\cdot\left[\frac{\sum_{j \in J}\left(\frac{\partial u^{h}\left(c_{t}^{h}, 1-l_{t}^{h}\right)}{\partial c_{j, h, t}^{i}} \cdot y_{j, h, t}\right)}{\sum_{j \in J} y_{j, h, t}}\right]^{-1}\left[\frac{\sum_{j \in J_{1}}\left(\frac{\partial u^{i}\left(c_{t}^{i}, 1-l_{t}^{i}\right)}{\partial c_{j, i, t}^{i}} \cdot y_{j, i, t}\right)}{\sum_{j \in J_{1}} y_{j, i, t}}\right]\right]^{-1}
\end{array}\right]
$$

para $i, h \in I, i \neq h$, y donde $y_{j, i, t}\left(y_{j, h, t}\right)$ es el producto nominal del sector $j$ en el país $i(h)$. Desde las ecuaciones (A.21) y (A.22) notamos que sólo $R E R_{t}$ depende del vector de ponderadores $a$. Betts y Kehoe (2001) demuestran la existencia de un único vector de ponderadores $a$, tal que el tipo de cambio real obtenido a partir del modelo del planificador mundial es equivalente con aquel obtenido mediante el modelo descentralizado. Más aún, los elementos del vector de ponderadores $a$ es proporcional al producto de los países, es decir, $\left(a^{i} / a^{h}\right) \propto\left(y_{j, i, t} / y_{j, h, t}\right)$.

En lugar de computar los elementos del vector de ponderadores $a$, el presente artículo estima el modelo del planificador mundial de tal forma que el estado estacionario de él replique los hechos estilizados observados en los datos del comercio bilateral entre Chile y Estados Unidos durante el período 1987-2007. Esto permitirá, finalmente, obtener un estimador $\hat{a}$ para el vector de ponderadores $a$. 


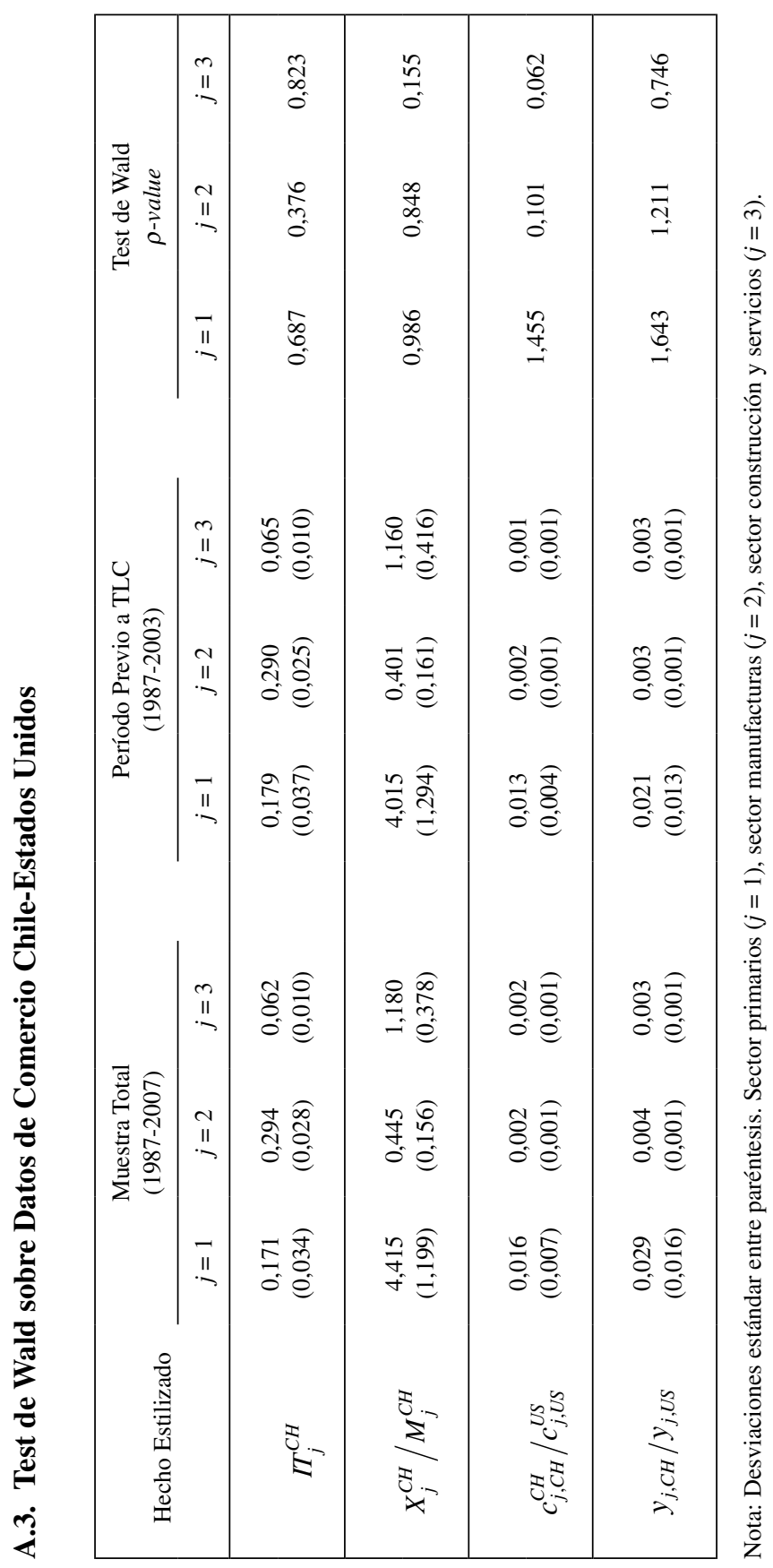


